\title{
Dimensional Crossover in the effective second harmonic generation of films of random dielectrics
}

\author{
P.M. Hui ${ }^{1}$, C. $\mathrm{Xu}^{2,1}$, and D. Stroud ${ }^{3}$ \\ ${ }^{1}$ Department of Physics, The Chinese University of Hong Kong, \\ Shatin, New Territories, Hong Kong \\ 2 Department of Physics, Suzhou University, Suzhou 215006, People's Republic of China \\ 3 Department of Physics, The Ohio State University, Columbus, Ohio 43210-1106
}

\begin{abstract}
The effective nonlinear response of films of random composites consisting of a binary composite with nonlinear particles randomly embedded in a linear host is theoretically and numerically studied. A theoretical expression for the effective second harmonic generation susceptibility, incorporating the thickness of the film, is obtained by combining a modified effective-medium approximation with the general expression for the effective second harmonic generation susceptibility in a composite. The validity of the theoretical results is tested against results obtained by numerical simulations on random resistor networks. Numerical results are found to be well described by our theory. The result implies that the effective-medium approximation provides a convenient way for the estimation of the nonlinear response in films of random dielectrics.
\end{abstract}

PACS Nos.: 42.65.An, 78.20.Bh 


\section{INTRODUCTION}

Nonlinear response of random composite materials has been a subject of intensive research for many years [1]. The nonlinear response in such composites may be affected by several factors. For example, the response can be greatly enhanced by local field effects, and the percolation effect could change the character of the system [2-9]. In particular, enhance-

ment of nonlinear optical response in random dielectrics may be useful for the design of novel switching devices in photonics and real-time coherent optical signal processors. A number of theoretical works have been developed specifically to weakly nonlinear composites [2-13]. An excellent review on the recent developments of the subject has recently been given by Shalaev [14].

While most of the work have focused on nonlinear effects such as the Kerr effect, Hui et al $[15,16]$ have derived general expressions for the effective susceptibilities for second harmonic generation ( $\mathrm{SHG}$ ) and third harmonic generation (THG) in a binary composite of random dielectrics. In the dilute limit, the effective SHG and THG susceptibilities obtained in Refs. [15] and [16] reduce to earlier expressions found by Levy et al. [17]. For many applications and materials fabrication, samples in the form of thin films are essential. It is, therefore, useful to study the effective SHG in films of random dielectrics. In the present work, we consider films of random dielectrics consisting of spherical particles with nonlinear response embedded randomly in a linear host. We study the dependence of the effective SHG on the film thickness and the concentration of the nonlinear component. By invoking a modified effective-medium approximation (EMA) to incorporate the film thickness in the estimation of the local fields, together with our previous general expression for SHG susceptibility, the effective SHG susceptibility including the effect of film thickness can be calculated. The validity of our theory is tested against results obtained by numerical simulations on random nonlinear resistor networks of different thickness consisting of two different kinds of resistors. It is found that our EMA provides a good description of numerical results for the whole range of thicknesses corresponding to systems spanning from two dimensions to three dimensions.

The plan of the paper is as follows. In Sec.II, we present our formalism on the effective SHG susceptibility of films of random dielectrics. Section III gives a description on the model of numerical simulations. Results of numerical simulations are compared with theoretical results in Sec.IV.

\section{EFFECTIVE MEDIUM APPROXIMATION FOR SHG IN FILMS}

We consider a macroscopically inhomogeneous medium consisting of a random mixture of two types of materials. The materials $a$ and $b$ have different macroscopic $\mathbf{D}$ - $\mathbf{E}$ relations. If we only include the quadratic nonlinearities, the general form of the $\mathbf{D}$-E relationship at zero frequency would be

$$
D_{i}=\sum_{j} \epsilon_{i j} E_{j}+\sum_{j k} d_{i j k} E_{j} E_{k}, \quad i=x, y, z
$$

where $D_{i}\left(E_{i}\right)$ is the $i$ th component of the displacement (electric) field $\mathbf{D}(\mathbf{E})$. For simplicity, we consider a composite in which a volume fraction $p$ of nonlinear material $a$ is mixed with a volume fraction $1-p$ of linear material $b$, i.e., $d_{i j k}(\mathbf{r})=d_{i j k}^{a}$ for regions occupied by material $a$ 
and $d_{i j k}(\mathbf{r})=0$ for regions occupied by material $b$. We also assume that the linear dielectric constants of both components are isotropic, i.e., $\epsilon_{i j}=\epsilon^{\alpha} \delta_{i j}$, with $\alpha=a(b)$ for regions occupied by material $a(b)$. In general, when a monochromatic external field is applied, the nonlinearity of the component inside the composite will generate local potentials and fields at all harmonic frequencies. Here, we focus on the effective second harmonic generation (SHG) for a thin of thickness $L$.

For a finite frequency external applied field of the form

$$
E_{0}(t)=E_{0, \omega} e^{-i \omega t}+\text { c.c. }
$$

the effective SHG susceptibility can be extracted by considering the volume average of the displacement field at the frequency $2 \omega$ in the inhomogeneous composite medium. A general expression for the effective SHG susceptibility has been derived in Ref. [15]. The result is that to first order in the SHG susceptibility $d_{i j k}$, the effective SHG susceptibility is given by $[15]$

$$
\left(d_{-2 \omega ; \omega, \omega}^{e}\right)_{i j k}=<K_{2 \omega ; i l} d_{l m n} K_{\omega, m j}^{T} K_{\omega, n k}^{T}>
$$

where $\langle\cdots\rangle$ denotes a volume average over the inhomogeneous medium. The tensors $K$ and $K^{T}$ are possible local field enhancement factors of the forms

$$
K_{2 \omega ; i l}(\mathbf{x})=\frac{E_{2 \omega ; l}(\mathbf{x})}{E_{0,2 \omega ; i}}
$$

and

$$
K_{\omega ; m j}^{T}(\mathbf{x})=\frac{E_{\omega ; m}(\mathbf{x})}{E_{0, \omega ; j}}
$$

with a similar expression for $K_{\omega ; n k}^{T}(\mathbf{x})$. The tensor $K_{\omega ; m j}^{T}(\mathbf{x})$, for example, is the induced $m$ th Cartesian component of the electric field at position $\mathbf{x}$ and frequency $\omega$ when a field $E_{0, \omega}$ is applied in the $j$-th direction at the same frequency in a linear random medium of the same spatial inhomogeneous structure. Similar expression for the effective THG susceptibility can also be derived [16].

In a composite in which only the material $a$ is nonlinear, the effective SHG susceptibility takes on the simple form

$$
\mathbf{d}_{-2 \omega ; \omega, \omega}^{e}=p \frac{<E_{2 \omega}^{a} \mathbf{d}_{-2 \omega ; \omega, \omega}^{a} E_{\omega}^{a} E_{\omega}^{a}>}{E_{0,2 \omega} E_{0, \omega} E_{0, \omega}} .
$$

It is desirable to further develop a simple analytic approximation for the average of the local field factors in Eq.(6). One such approximation is the effective medium approximation (EMA). One possible effective medium approximation is to calculate the electric fields within each particle as if that particle is embedded in an effective medium with dielectric constant $\epsilon^{e}[16]$. In this approximation, Eq.(6) becomes

$$
\mathbf{d}_{-2 \omega ; \omega, \omega}^{e}=p \mathbf{d}_{-2 \omega ; \omega, \omega}^{a}\left(\frac{D \epsilon_{2 \omega}^{e}}{\epsilon_{2 \omega}^{a}+(D-1) \epsilon_{2 \omega}^{e}}\right)\left(\frac{D \epsilon_{\omega}^{e}}{\epsilon_{\omega}^{a}+(D-1) \epsilon_{\omega}^{e}}\right)^{2},
$$


where $D$ is the spatial dimension and the subscripts $\omega$ and $2 \omega$ specify the frequency at which the local field is evaluated.

The effective linear dielectric constants $\epsilon_{\omega}^{e}$ and $\epsilon_{2 \omega}^{e}$ can be evaluated from the usual EMA for linear random composites by

$$
\sum_{\alpha=a, b} p_{\alpha}\left(\frac{\epsilon^{\alpha}-\epsilon^{e}}{\epsilon^{\alpha}+(D-1) \epsilon^{e}}\right)=0
$$

where $p_{\alpha}$ is the volume fraction of the $\alpha$ th component and the summation is over the different components in the composite. For a thin film of thickness $L$, the behaviour of the system lies somewhere between $D=2$ for small thickness and $D=3$ for large thickness. To incorporate the effects of finite thicknesses, it is most convenient to convert the continuum description into a discrete version of the problem. In the discrete version, the system is considered to be a lattice consisting of $N \times N \times L$ sites, where $L$ is the number of layers. For a $D$-dimensional hypercubic lattice, the coordination number $z$, i.e., the number of nearest neighbors, is related to $D$ through $D=z / 2$. For a single layer $(L=1), z=4$ and for a large number of layers $(L \rightarrow \infty)$, the system is three dimensional with $z=6$. For a system of finite thickness $L$, the average coordination number becomes $L$-dependent and takes on

$$
z(L)=2\left(3-\frac{1}{L}\right)
$$

Incorporating Eq.(9) into Eqs.(7) and (8) by replacing the dimensionality $D$ by $z(L) / 2$ constitute a set of equations for a modified EMA for the effective SHG susceptibility of films of random dielectrics.

\section{NUMERICAL SIMULATIONS USING RANDOM NONLINEAR RESISTOR NETWORK}

To test the validity of the modified EMA for SHG susceptibility in a film of random dielectrics, we follow the approach in Ref. [16] and perform numerical simulations using a random nonlinear resistor network. Such an approach has been applied previously with success to study linear optical properties and nonlinear properties such as the Kerr effect in random composites [18-21]. To model a system of finite thickness, two types of bonds are placed randomly onto a $N \times N \times L$ lattice, with $L$ running from 1 to $N$ modelling a 2D system $(L=1)$ and 3D system $(L=N)$, respectively. In the resistor network model, it is more convenient to define the model in terms of conductance of the bonds. With probability $p$, the bonds in the lattice are occupied by nonlinear components characterized by the two parameters: the linear conductance $g^{a}$ and the nonlinear susceptibility $d$, with the latter representing the response to two voltages at frequency $\omega$. With probability $(1-p)$, the bonds are occupied by linear components characterized by a linear conductance $g^{b}$. To model a mixture of metallic and insulating components at finite frequencies, we take $g^{a}$ and $g^{b}$ to be of the forms

$$
g^{a}(\omega)=\frac{1+i \omega R C-\omega^{2} L C}{R+i \omega L}
$$


and

$$
g^{b}(\omega)=i \omega C
$$

The nonlinear susceptibility $d$ of material $a$ is assumed to be independent of frequency, for simplicity. This choice of $g^{a}$ and $g^{b}$ has been widely used in studying the frequency response in random composites $[16,20,21]$, because the ratio $g^{a} / g^{b}$ has the same form as the Drude

dielectric function of a metal with the plasma frequence $\omega_{p}=\sqrt{1 / L C}$ and relaxation time $\tau=L / R$. In what follows, the parameters are chosen to be $C=1, L=1$, and $R=0.1$, corresponding to a plasma frequency $\omega_{p}=1$.

The effective response of the random nonlinear resistor network can be studied by direct numerical simulations [16]. Using the Kirchhoff's law, the voltage at each node can be solved. Although the applied voltage imposed on the nonlinear network is taken to consist only of a component at frequency $\omega$, the voltage at each node inside the network has components at frequencies $\omega$ and $2 \omega$. So, solving the Kirchhoff's law leads to solving two equations $\sum I^{(\omega)}=0$ and $\sum I^{(2 \omega)}=0$ at each node simultaneously, where the summation is over all the bonds connected to the node under consideration and $I^{(\omega)}$ and $I^{(2 \omega)}$ are the component of the current corresponding to the frequency $\omega$ and $2 \omega$, respectively. These two equations at each node must be solved self-consistently for the voltages at frequencies $\omega$ and $2 \omega$, and the effective SHG can be then extracted. In what follows, we use a lattice of size $20 \times 20 \times L$, with an applied voltage set to unity. By varying the layer thickness $L$, dimensional crossover of the effective SHG response from 2D to 3D can be studied.

\section{RESULTS AND DISCUSSION}

Numerical simulations allow for the extraction of the effective linear and nonlinear response of the inhomogeneous system for different concentrations of the nonlinear component and for different film thicknesses. Results from numerical simulations can then be compared with those calculated via the modified EMA so as to establish the validity of the approximation. Figure 1 and Figure 2 show the effective linear response $g_{\text {eff }}$ and effective SHG response $d_{e} / d$ of the random nonlinear network as a function of frequency $\omega$ for three values $(p=0.1,0.5,0.9)$ of the concentration $p$. In each panel of the figures, the symbols give the simulation results and the lines give the EMA results. The symbols represent an average over 50 independent runs corresponding to different configurations of the random network. The EMA results are obtained by using Eqs.(7)-(9), with the dielectric constants replaced by the conductances. Four different layer thicknesses are studied for each value of the concentration.

In the dilute case $(p=0.1)$, the concentration is lower than the percolation threshold regardless of the thickness, i.e., $p<p_{c}$ for both $p_{c}(2 D)$ and $p_{c}(3 D)$ in the bond percolation problem. In this case, isolated nonlinear bonds (component $a$ ) are surrounded by the insulating bonds (component $b$ ). In the continuum case, the situation corresponds to one in which isolated disks (or spheres) of component $a$ are embedded in a background of component $b$. The structure observed in $g_{\text {eff }}$ corresponds to the so-called surface plasmon resonance structure. The surface plasmon resonance peak shifts to lower frequency as the layer thickness increases. The shift is related to the change in the resonance frequency from 
$\omega_{p} / \sqrt{3}$ for an isolated sphere in 3D (large $\left.L\right)$ to $\omega_{p} / \sqrt{2}$ for an isolated disk in $2 \mathrm{D}(L=1)$. The real and imaginary parts of the nonlinear response normalized to the SHG coefficient of the nonlinear component $d_{e} / d$ show a corresponding resonance structure near the surface plasmon resonance frequency. The modified EMA captures the features reasonably well, with a better agreement for large values of $L$. It is reasonable in that the EMA, being a mean field approximation, is expected to work better in high spatial dimensions in which fluctuation effects are smaller. We also note that the simulation results indicate that the system picks up three dimensional character readily for not-so-large layer thicknesses.

For $p=0.5$, which is the percolation threshold for an infinite 2D system and higher than $p_{c}(3 D)$, the numerical results for $\operatorname{Re}\left(g_{\text {eff }}\right)$ with $L=1$ show a broad structure with the values at low frequencies tend to be enhanced. For $L \geq 2$, large $\operatorname{Re}\left(g_{\text {eff }}\right)$, relative to the dilute case, at low frequencies indicates that the composite behaves effectively as a conducting (metallic) system. The greater the thickness, the larger is $\operatorname{Re}\left(g_{\text {eff }}\right)$. It is because the value of $p=0.5$ becomes increasingly larger than $p_{c}$ as $L$ increases, and the system shows increasingly dominating metallic character. The effective SHG response shows a broader structure with an enhanced $d_{e} / d$ compared with the dilute concentration case. The enhancement in the SHG response comes about from the enhancement in the local field applying on the nonlinear component when the system has more better conducting nonlinear component. The modified EMA, again, gives results in reasonable agreement with simulation results, indicating that incorporating a thickness-dependent coordination number into EMA can be used to estimate the effective SHG response in random dielectric systems.

For high concentrations $(p=0.9)$ of the nonlinear component, the systems shows metallic behavior in the linear response, as shown in Fig.1. The behavior of the effective SHG susceptibility basically follows that of the case of $p=0.5$, with the appearance of a small resonance structure at low frequency. The structure is due to the isolated insulating component in an otherwise metallic background.

In both our numerical and EMA results, the SHG composite susceptibility is enhanced only relatively weakly over that of the pure SHG material. By contrast, such processes as Kerr nonlinearity are greatly enhanced in composite media [2-5,7-13,22,23]. We believe this weak enhancement may occure because Eq. (6) involves the linear fields at two different frequencies. Thus, the enhancement will be smaller than when all the field enhancement factors are resonant at the same frequency. The enhancement is further weakened by randomness when both components are present in high concentrations, which causes the field enhancement factors to be broadened over a range of frequencies. At very low concentrations, despite the two frequencies, other studies have shown that the SHG enhancement is still large.

Also, our calculations assume that only the metallic component has a nonzero SHG susceptibility. We have done a few test EMA calculations in 2D, and find a somewhat larger enhancement, when only the nonmetallic component is nonlinear, with $\operatorname{Im}\left(d_{\text {eff }} / d\right)$ and $\operatorname{Re}\left(d_{e f f} / d\right)$ reaching magnitudes as large as 4 or 5 .

Expression (6) for the effective SHG susceptibility treats the composite as a homogeneous medium with effective properties, as is reasonable if the inhomogeneities are small compared to a wavelength. Since the medium is effectively homogeneous, the SHG emission will be in the form of a collimated beam. Besides this beam, there is another SHG contribution, resulting from scattering from the inhomogeneities. This contribution, which has a broad 
angular distribution, depends on particle size, unlike that calculated here. It has been seen in experiments on inhomogeneous metal-insulator films [24], and may be large near the percolation threshold.

\section{SUMMARY}

We have studied the dependence of the effective SHG response in a random dielectric system as a function of the concentration of the nonlinear constituent and the systemn thickness. A modified EMA, based on a previous expression of the effective SHG derived by the authors coupled with a thickness-dependent coordination number, is proposed for the effective SHG response. Numerical simulations on a random network consisting of a mixture of nonlinear and linear conductances allow extraction of the effective linear and SHG response. It is found that the modified EMA gives results that are in reasonable agreement with the numerical simulation results. The system picks up three dimensional character for moderate film thicknesses. The results show that the modified EMA can be used as an effective tool for estimating the effective SHG response in random dielectric systems. The approximation can be applied to other systems consisting of nonlinear components. For example, the nonlinear SHG response may be associated with the poorer conductor of the two constituents. In this case, the contrast in the linear dielectric constants between the two constituents may produce an enhanced local field in the poorer conducting component and thus drive a greater SHG enhancement. Indeed, some preliminary calculations, using the EMA, do give rise to this increased enhancement.

\section{ACKNOWLEDGMENTS}

This work was supported in part by a grant from the Research Grants Council of the Hong Kong SAR Government through the grant CUHK4129/98P. One of us (DS) acknowledges support from the National Science Foundation through the grant DMR01-04987. 


\section{REFERENCES}

[1] V. M. Shalaev, Phys. Rep. 272, 61 (1996); D. J. Bergman and D. Stroud, Solid State Physics 46, 147 (1992); C. Flytzanis, F. Hache, M. C. Klein, and P. Roussignol, in Progress in Optics 29, edited by E. Wolf (North-Holland, Amsterdam, 1991), p. 322.

[2] D. Stroud and Van E. Wood, J. Opt. Soc. Am. B 6, 778 (1989).

[3] A. E. Neeves and M. H. Birnboin, J. Opt. Soc. Am. B 6, 787 (1989); Y. Q. Li, C. C. Sung, R. Inguva, and C. M. Bowden, ibid, 6, 814 (1989); J. W. Haus, N. Kalyaniwalla, R. Inguva, M. Bloemer, and C. M. Bowden, ibid, 6, 797 (1989).

[4] P. M. Hui, Phys. Rev. B 41, 1673 (1990); 4915344 (1994).

[5] D. Stroud and P. M. Hui, Phys. Rev. B 37, 8719 (1988).

[6] A. Aharony, Phys. Rev. Lett. 58, 2726 (1987).

[7] J. W. Haus, R. Inguva, and C. M. Bowden, Phys. Rev. A 40, 5729 (1989).

[8] O. Levy and D. J. Bergman, Phys. Rev. B 46, 7189 (1992).

[9] V. M. Shalaev, E. Y. Poliakov, and V. A. Markel, Phys. Rev. B 53, 2437 (1996) and references therein.

[10] X. C. Zeng, D. J. Bergman, P. M. Hui, and D. Stroud, Phys. Rev. B 38, 10970 (1988).

[11] P. M. Hui, J. Appl. Phys. 68, 3009 (1990).

[12] R. Levy-Nathansohn and D. J. Bergman, J. Appl. Phys. 77, 4263 (1995).

[13] J. E. Sipe and R. W. Boyd, Phys. Rev. A 46, 1614 (1992); R. W. Boyd and J. E. Sipe, J. Opt. Soc. Am. B 11, 297 (1994).

[14] V. M. Shalaev, Nonlinear Optics of Random Media: fractal composites and metaldielectric films (Springer, New York 2000); see also V. M. Shalaev (ed.), Properties of nanostructured random media (Springer, New York, 2002).

[15] P. M. Hui and D. Stroud, J. Appl. Phys. 82, 4740 (1997).

[16] P. M. Hui, P. Cheung, and D. Stroud, J. Appl. Phys. 84, 3451 (1998).

[17] O. Levy, D. J. Bergman, and D. G. Stroud, Phys. Rev. E 52, 3184 (1995).

[18] X. Zhang and D. Stroud, Phys. Rev. B 52, 2131 (1995).

[19] P. M. Hui, W. M. V. Wan, and K. H. Chung, Phys. Rev. B 52, 15867 (1995).

[20] R. S. Koss and D. Stroud, Phys. Rev. B 35, 9004 (1987).

[21] X. C. Zeng, P. M. Hui, and D. Stroud, Phys. Rev. B 39, 1063 (1989).

[22] H. Ma, R. Xiao and P. Sheng, J. Opt. Soc. Amer. B15, 1022 (1998).

[23] A. K. Sarychev and V. M. Shalaev, Physics Reports 335, 275 (2000).

[24] M. Breit, V. A. Podolskiy, S. Grésillon, G. von Plessen, J. Feldmann, J. C. Rivoal, P. Gadenne, A. K. Sarychev, and V. M. Shalaev, Phys. Rev. B64, 125106 (2001). 


\section{FIGURES}

FIG. 1. The effective linear response $g_{\text {eff }}$ as a function of $\omega$. The three panels correspond to $p$ $=0.1,0.5$, and 0.9. In each panel, results for thickness $L=1,2,4$, and 6 are shown. The symbols are results of numerical simulations and the curves are theoretical results based on the EMA.

FIG. 2. The effective SHG response as a function of $\omega$. The three panels correspond to $p=$ 0.1, 0.5, and 0.9. In each panel, results for thickness $L=1,2,4$, and 6 are shown. The symbols are results of numerical simulations and the curves are theoretical results based on the EMA. 


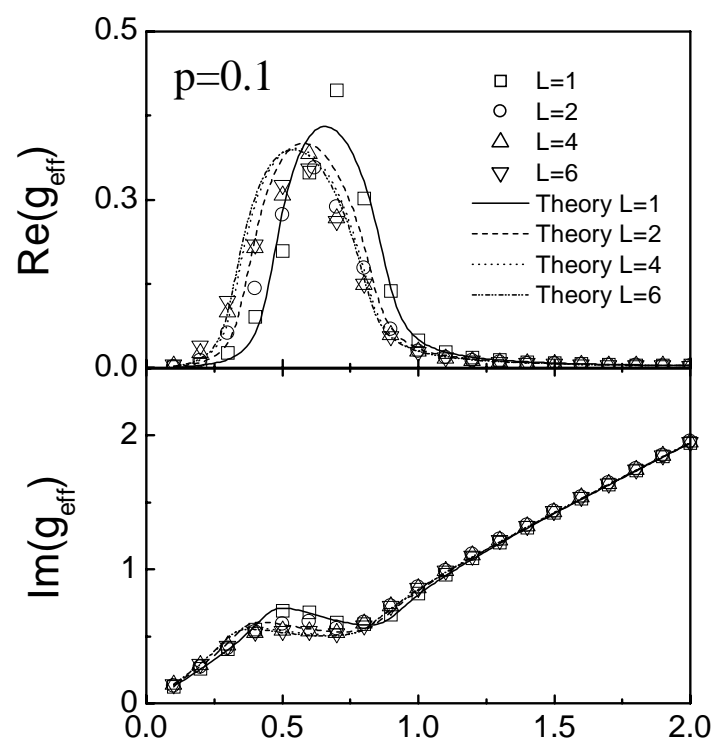

$\omega$

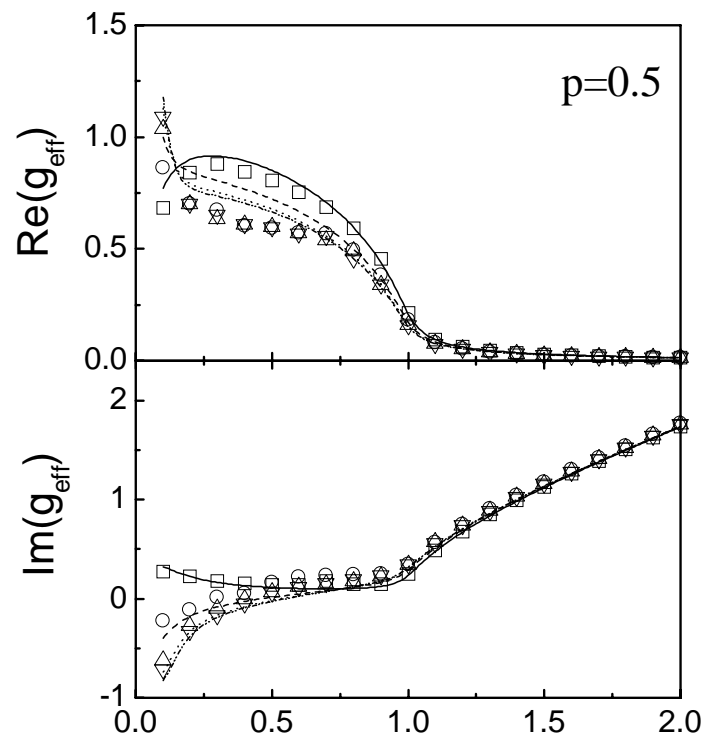

$\omega$

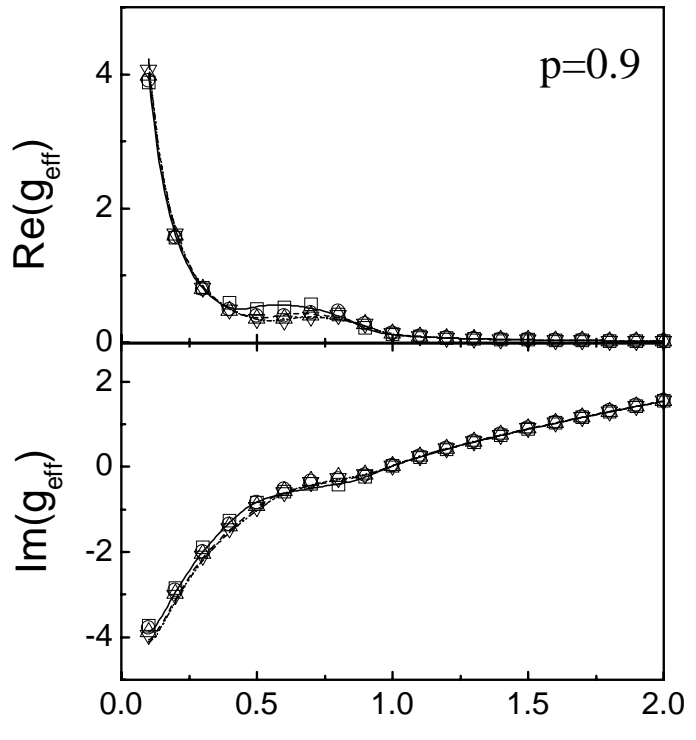

$\omega$

Figure 1 


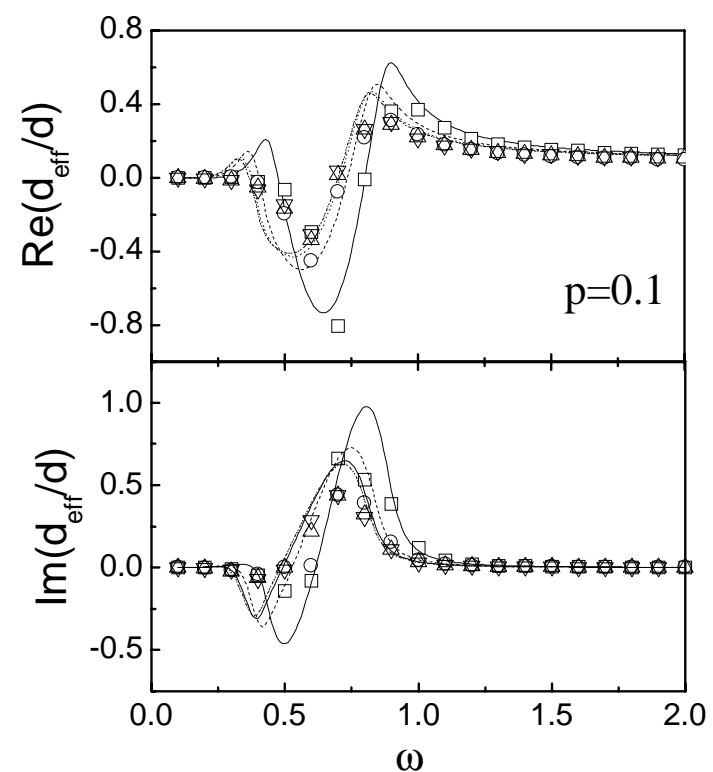

ㅁ $\mathrm{L}=1$

ㅇ $\mathrm{L}=2$

$\triangle \mathrm{L}=4$

$\nabla \quad \mathrm{L}=6$

Theory $L=1$

Theory $L=2$

Theory $L=4$

Theory $L=6$

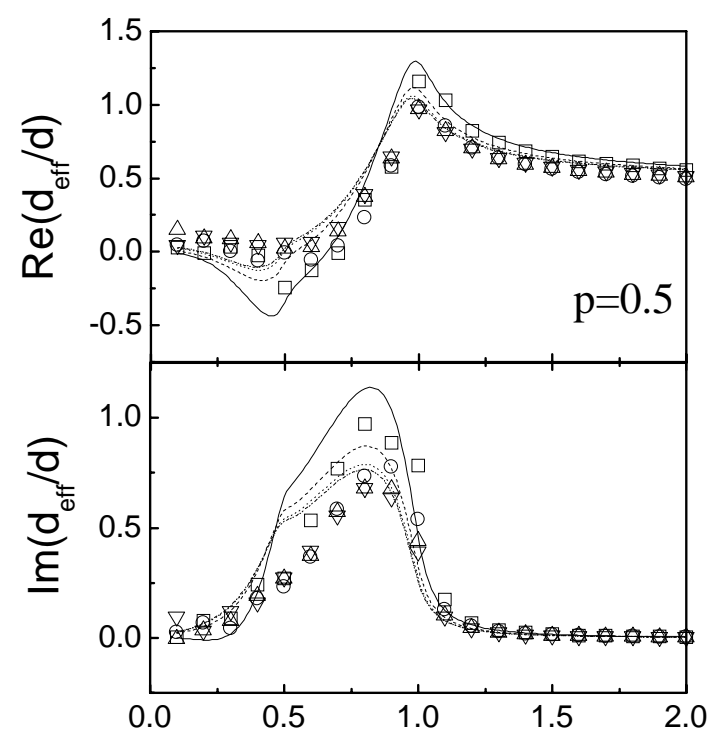

$\omega$

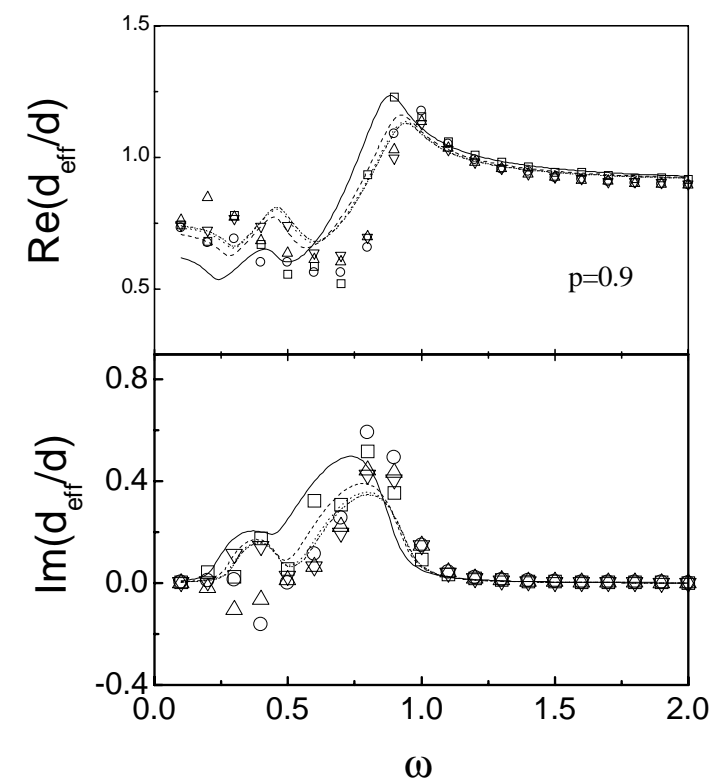

Figure 2 